\title{
Efficacy of an Asthma Self-management Education Intervention for Children (9-13 Years) with Asthma and Their Caregiver in Wuhan, China
}

\author{
Zhenghui Liu ${ }^{1}$ and Kristine Qureshi ${ }^{2}$ \\ 1. HOPE Nursing School of Wuhan University, Wuhan, Hubei 430061, China \\ 2. School of Nursing and Dental Hygiene, University of Hawaii, Manoa, Hawaii 96844, USA
}

\begin{abstract}
OBJECTIVE: Evaluate the efficacy of an asthma self-management education program delivered to children and their caregiver at an outpatient clinic in Wuhan, China. METHOD: A quasi-experimental, two-group, pretest/posttest design was used. A convenience sample of children with asthma $(N=81)$ and their caregiver completed a survey at baseline and at 2-month follow-up. The experimental group $(n=41)$ received a 45-minute cognitive-behavioral asthma self-management educational intervention with 3 follow-up contacts at 15 -day intervals before their scheduled revisit to the clinic at 2 months post-intervention. The control group $(n=$ 40) received the current standard of care. RESULTS: No statistically significant differences were found in the groups at baseline based on demographic or disease characteristics or outcome measures. Compared to participants in the control group, participants in the intervention group demonstrated statistically significant improvement $(P<0.05)$ in asthma self-management, frequency of peak flow meter use, asthma control, and child and caregiver quality of life. CONCLUSION: The outpatient asthma self-management education intervention was deemed efficacious for children with asthma and their caregiver.
\end{abstract}

Key words: Self-management education, children, caregiver, parent, asthma control, quality of life, pulmonary function.

\section{Introduction}

Asthma is a global health problem affecting about 300 million people across all ages, ethnic groups and countries. It is considered to be one of the most common chronic childhood health conditions [1]. Children with asthma and their caregivers/parents report physical, economical, and psychological burdens associated with asthma [2-4]. Over 9\% of children in the United States (6.7 million) were diagnosed with asthma in 2007 and its prevalence continues to increase [5]. The prevalence rate of childhood asthma in urban areas of China increased from $1.97 \%$ in 2000 to $2.32 \%$ in 2013 [6]. Asthma severity and inadequate symptom control are serious problems, especially in developing countries [7].

Corresponding author: Kristine Qureshi, Ph.D., RN, FAAN, CEN, APHN-BC, professor, program director, research fields: epidemiology, medical science nursing, and disaster medicine.
Insufficient knowledge about prevention and treatment, misunderstanding about steroid therapy, irregular use of medications and treatments, and unsatisfactory or ineffective communication with health care providers exists widely around the world [8].

Education about self-management as an effective intervention to improve the outcomes of asthma has been recommended [7]. Studies focusing on childhood asthma education vary extensively in different areas. Asthma education programs are conducted at home, in the community, in schools, clinics, and hospital settings [9-13]. Most studies assessed asthma management outcomes by recording the rate of emergency room visits and hospitalization, school absenteeism, caregiver/parent's work days missed, and quality of life $[10,11,13,14]$. Few studies have used objective indicators such as the ratio of forced expiratory volume in 1 second (FEV1\%), which reflects changes in pulmonary function over time. 
Repeat pulmonary function assessments should be considered when evaluating the efficacy of an asthma management program in children [2].

In China, the education of children regarding asthma management is not considered a standard of care. In this study, an outpatient asthma self-management intervention was used to address health disparities in asthma education for children and their caregivers/parents, hereafter referred to as caregivers. The purpose of this study was to evaluate the efficacy of an outpatient asthma self-management education intervention for children with asthma and their caregiver in Wuhan, China. Independent variables included a diagnosis of asthma, the age and gender of the child, urban or rural residence, family structure, the caregiver's level of education, family history of asthma, and disease duration. Intermediate outcome variables included asthma self-management and frequency in the use of a peak flow meter (PFM). Asthma self-management was defined as the child's ability to assess and monitor asthma related symptoms, use medication as recommended, and to maintain a balance in psycho-social adaption, and included frequency in use of the PFM. Long-term outcome variables included asthma control, pulmonary function, and the quality of life of the child and caregiver. Asthma control was defined as "the full range of clinical impairment that patients with asthma may experience as a result of their disease" [15]. Pulmonary function was assessed using FEV1\%. Quality of life was assessed using the Standardized Pediatric Asthma Quality of Life Questionnaire (PAQLQ) and the Pediatric Asthma Caregiver's Quality of Life Questionnaire (PACQLQ).

The research question asked, what is the efficacy of a new outpatient asthma self-management education intervention for children with asthma and their caregiver? Compared to participants in the control group who receive standard care, children and caregivers who received the educational intervention were hypothesized to demonstrate increased asthma self-management, frequency of use of the PFM, improved asthma control, FEV1\%, and child and parent quality of life.

\section{Methods}

\subsection{Design}

A quasi-experimental, two-group, pretest/posttest design was used to evaluate the efficacy of the asthma self-management education intervention. The institutional review board of Wuhan HOPE School of Nursing and Wuhan Children's Hospital approved this study. The principle investigator (PI) conducted this study as a requirement for a Master of Science degree in Nursing after completing an intensive internship with medical and nursing asthma specialists. The PI was solely responsible for participant recruitment and enrollment, and program delivery including follow-up contacts as well as data collection and analysis. After being informed of the purpose and aims of the study, roles and responsibilities of participants, and risks and benefits to be derived, written consent for the self and permission for his/her child to participate was obtained from the children and their caregivers. No unanticipated events were reported.

\subsection{Sample, Sampling and Sample Size}

A convenience sample of children, aged 9-13 years with intermittent and mild to severe persistent asthma, and their caregiver was recruited from Wuhan Children's Hospital Outpatient Department. The outpatient department with 3 physicians annually serves approximately 600 children with asthma residing in urban and rural areas of central China average year.

Power analysis was conducted based on outcome measures with a clinically significant projected effect size of 0.35 ( $\alpha: 0.05, \beta: 0.8)$. A sample size of 68 families at the 2-month follow-up visit was considered sufficient across all outcome measures.

\subsection{Participant Eligibility, Recruitment and Enrollment}

Participants were recruited and enrolled based on 

with Asthma and Their Caregiver in Wuhan, China

specific inclusion and exclusion criteria. Child inclusion criteria included: (1) a diagnosis of asthma as defined in Practical Pediatrics in 2008 revised edition [16], (2) between the age of 9 to13 years at the time of enrollment, (3) caregiver report of age-appropriate cognitive functioning in his/her child, (4) caregiver informed written consent, and (5) child verbal and written consent. Child exclusion criteria included: (1) the presence of one or more respiratory system diseases except asthma, (2) caregiver report of the presence of cognitive impairment in child that was significantly below age level, or (3) lack of verbal and written consent and caregiver permission. Caregiver inclusion criteria included: (1) being the caregiver of a child diagnosed with asthma and (2) informed verbal and written consent. Caregiver exclusion criteria included self-report of a mental health condition such as clinical depression that might alter perceptions of one's quality of life.
Participants were recruited during scheduled outpatient department visits. Caregivers of children with a diagnosis of asthma based on medical record data were offered a recruitment flier. Of the 89 families who met inclusion criteria and enrolled in the study, 81 completed baseline surveys. Participants were divided into one of two groups based on the child's medical record number. Participants with odd numbers $(n=41)$ were assigned to the treatment group and received the intervention. Participants with even numbers $(n=40)$ were assigned to the control group and received routine care. The CONSORT chart contains enrollment, group assignment, participation, and 2-month follow-up data. See Fig. 1.

\subsection{Intervention}

Child and caregivers participated in one 45-minute face-to-face asthma management teaching session conducted in the outpatient department meeting room

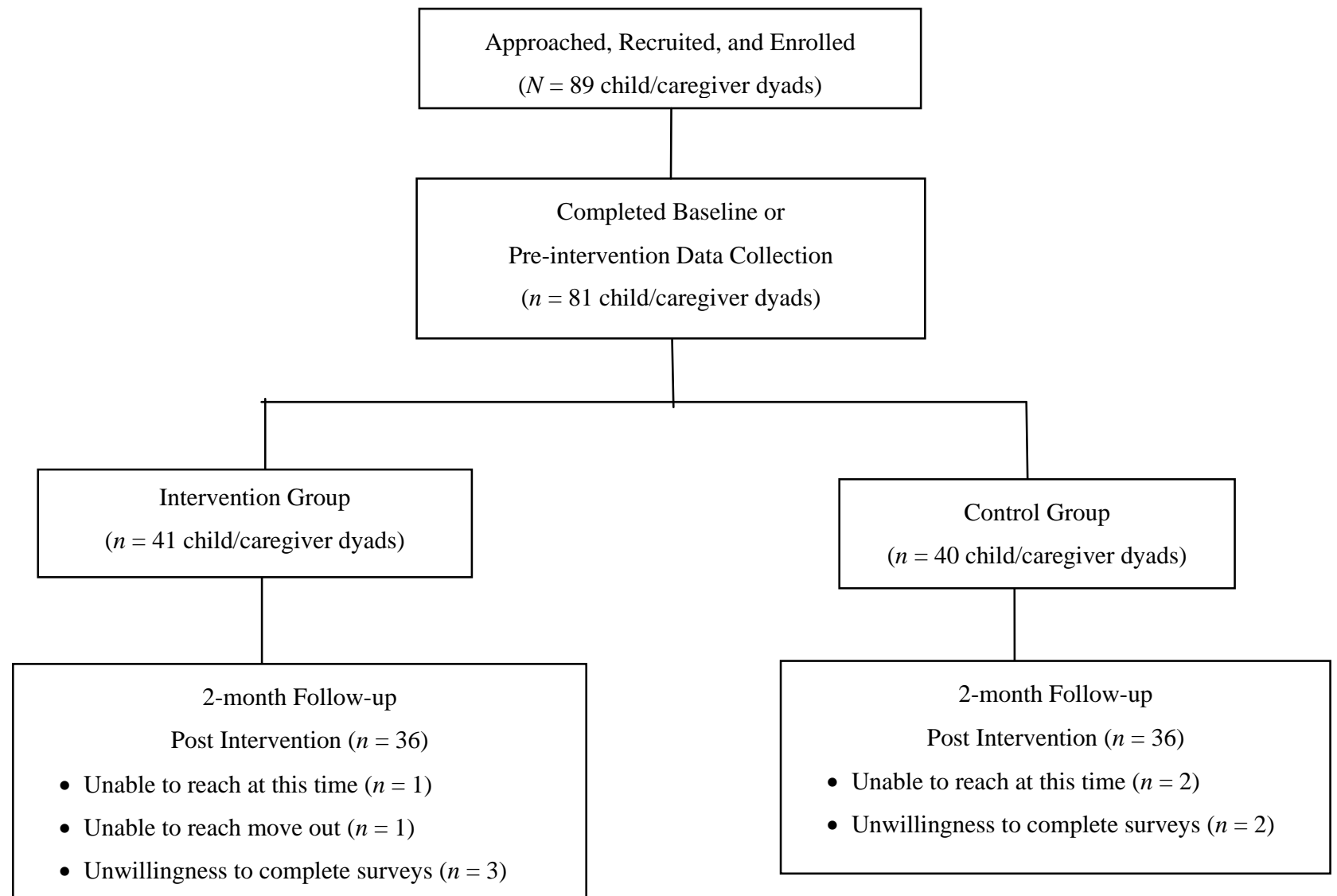

Fig. 1 CONSORT chart. 
immediately following a clinic visit during which pulmonary function tests were performed. The purpose of the class was to share recommendations of the Global Initiative for Asthma and National Heart, Lung, and Blood Institute [7, 16, 17]. Teaching was delivered by lecture, discussion, demonstration, and return demonstration formats according to the following outline: (1) introduction of pediatric asthma including recognition of asthma symptoms, assessment of asthma control, and clarification of common misunderstandings about pediatric asthma; (2) discussion of asthma therapies including pharmaceutical and non-pharmaceutical treatments and safety precautions to consider when using prescriptions for inhaled and oral steroids; (3) demonstration on proper use of three types of inhaler devices; (4) control of common indoor and outdoor environmental triggers or stimuli by reducing exposure; (5) discussion of self-monitoring strategies specifically use of the PFM and a diary to record asthma symptoms and management activities; (6) recognition of asthma exacerbation using the PFM and symptom diary.

Outcome learner objectives specified that by completion of the intervention session, participants would be able to (1) recognize asthma symptoms, (2) evaluate the level of asthma control, (3) demonstrate proper use of the inhaler, (4) identify and control the stimuli or triggers, (5) monitor asthma symptoms, (6) use the PFM, and (7) recognize acute exacerbation of symptoms. Proper use of the PFM and prescribed inhaler was demonstrated and practiced to achieve proficiency. Each family received a PFM and diary for recording asthma symptoms and management activities.

WeChat, a free messaging application for mobile phones, allowed caregivers the opportunity to share information with the nurse researcher and for the nurse researcher to address any questions about the child's asthma management. In addition, three telephone follow-up calls were made at approximately 15-day intervals after delivery of the educational session.
Information about medication compliance, asthma symptoms, use of the PFM, and content contained in the asthma diary was reviewed and questions were answered. Participants were seen in the outpatient department for the final follow-up session during their clinic visit approximately two months after baseline data collection and delivery of the program. A follow-up FEV1\% was obtained during the clinic visit.

The control group received routine care in the outpatient department that consisted of a one-way health education session using a manual about asthma management without any further interaction. Participants were seen in the outpatient department approximately two months after baseline data collection. The follow-up FEV1\% was obtained during the clinic visit. After completing the final data collection session, the children and their caregivers initially assigned to the control group were offered and participated in the asthma self-management education intervention described above.

\subsection{Data Collection}

A structured interview was used for data collection. Prior to data collection, all survey questionnaires items were reviewed by three children, two males and one female, in the target age range who were asked to complete a questionnaire independently. The researcher then reviewed each item in the questionnaire and asking each child what he/she thought the item meant. The children were able to complete the questionnaire with understanding and comprehension of the content.

Participants completed a series of instruments deemed reliable and valid for the target population. Children completed the Pediatric Asthma Self-management Scale (PASMS), the Asthma Control Questionnaire (ACQ), and the Standardized Pediatric Asthma Quality of Life Questionnaire (PAQLQ) and reported on their use of the PFM. Caregivers completed a demographic survey and the Pediatric Asthma Caregiver's Quality of Life Questionnaire (PACQLQ). 


\subsection{Demographic Survey}

Caregivers completed an 8-item survey created by the PI to obtain demographic and disease related information. Demographic information included child's age, gender, residence, number of siblings, family structure, and caregiver's educational level. Disease-related information included family asthma history and disease duration.

\subsubsection{Pediatric Asthma Self-management Scale (PASMS)}

This is a 34 item, 5-point Likert type scale with three subscale developed by Hu Ping in 2010 [18]. It measures the level of self-management of children with asthma. The sub scales captured management related to daily living, medication usage and psycho-social considerations. Response options range from always (every day), usually (4-6 times a week), sometimes (2-3 times a week), occasionally (1-2 times a week), to never. Negatively worded items are reverse scored. Scores are summed with a potential range from 34 to 170 with higher scores reflecting better self-management. Cronbach's $\alpha$ reported by the developer for the entire scale was 0.87 , split-half-reliability was 0.90 and the test-retest reliability was 0.87 [18]. Internal consistency based on Cronbach's $\alpha$ for this sample was 0.80 .

A peak flow meter (PFM) is a device that predicts changes in airway obstructions by (1) assessing the severity of asthma exacerbation, (2) detecting early stages of asthma episodes, (3) monitoring response to medication, and (4) establishing an objective measure for detecting asthma triggers or stimuli. Frequency of PFM use was measured by a single item indicator asking: How often do you use your PFM? Response options ranged from $0=$ never to $1=$ occasionally $(1-2$ times a week), 2 = sometimes (2-3 times a week), $3=$ usually (4-6 times a week), and $4=$ always (every day).

\subsubsection{Asthma Control Questionnaire (ACQ),} Chinese Version

This is a 7-item, 7-point Likert type scale developed by Juniper [19] that was later translated by Dr. Yu-zhi Chen and Dr. Jin-zhunWu with the assistance of
Juniper and the MAPI Research Institute into Chinese. The scale captures both adequacy and changes in asthma control. Items assess level of impairment based on waking in the night due to symptoms, waking in the mornings with symptoms, limitation of daily activities, shortness of breath, and wheezing, as well as two items recording FEV1\% along with use of long-acting and short-acting inhaled bronchodilators. Participants were asked to recall the status of their asthma during the previous week before responding to each item. Response options range from 0 indicating no impairment to 6 indicating maximum impairment. Scores were averaged and had the potential range from 0 to 6 with higher scores indicating maximum impairment. As the developers recommend a cut point of in clinical trials as indicating a participant is well-controlled. Cronbach's $\alpha$ for this sample was 0.77 .

2.6.3 Percent of Forced Expiratory Volume in One Second Predicted FEV1\%

FEV1\% was endorsed by the National Asthma Education and Prevention Program (NAEPP) to assess asthma severity and predict important disease outcomes [20]. Pulmonary function tests (PFT) for participants in this study were performed by trained personnel working in the respiratory disease department of Wuhan Children's Hospital of China. Results were obtained from these personnel.

2.6.4 Standardized Pediatric Asthma Quality of Life Questionnaire, Chinese Version (PAQLQ(S))

This is a 23-item, 7-point Likert type scale developed by Juniper [21] and later revised from the original version [22] by Juniper and MAPI Research Institute for use with Chinese populations. The scale assesses physical, emotional and social functional problems identified as most troublesome to children with asthma. Items in the scale capture three domains: activity limitation (5 items), symptoms (10 items), and emotional function ( 8 items). Children were asked to reflect on how they were affected by asthma during the previous week before responding to each item. Response options ranged from 1 = extremely bothered to $7=$ not bothered at all. Scores were summed and had 
the potential range from 1 to 7 . Cronbach's $\alpha$ for this sample was 0.88 .

2.6.5 Pediatric Asthma Caregivers Quality of Life Questionnaire (PACQLQ), Chinese Version

This 13-item, 7-point Likert type scale was developed by Juniper in 1996 to measures problems that are most troublesome to caregivers of children with asthma. The scale captures two domains: activity limitation and emotional function. Caregivers were asked to recall the impact that their child's asthma had on their quality of life during the previous week. Response options ranged from 1 (extremely bothered) to 7 (not bothered at all). Scores were summed and had the potential to range from 1 to 7 with higher scores reflective of higher quality of life. The scale has demonstrated reliability, with an intra-class correlation coefficient for overall quality of life, activity limitation, and emotional function of $0.85,0.84$, and 0.80 respectively [23]. Using the overall quality of life scale that included all items, Cronbach's $\alpha$ for this sample was 0.87 .

\subsection{Data Analysis}

SPSS 19.0 (IBM SPSS, 2010) was used for data entry, management, and analysis. Descriptive statistics were computed for all variables. Non-parametric and parametric tests were used to analyze the data. Chi-square was used to determine whether baseline differences based on categorical demographic data existed between the intervention and control groups. A paired sample t-test was used to determine if there was a statistically significant improvement $(P<0.05)$ from pre-intervention to 2-months post-intervention in the intervention group and in the control group based on asthma self-management, frequency of PFM use, asthma control, FEV1\%, and child and caregiver quality of life. The independent sample t-test was used to determine if there was a statistically significant difference $(P<0.05)$ between the intervention and control group based on the same variables at the 2-month post-intervention follow-up clinic visit.

\section{Results}

\subsection{Demographic Characteristics}

Participants included children aged 9-13 years $(\mathrm{M}=$ $9.89, \mathrm{SD}=0.80$ ). A total of $72 \mathrm{child} /$ caregiver dyads completed the study with equal numbers $(n=36)$ in both the intervention and control groups. Demographic characteristics are presented in Table 1. Children under age 10 years comprised $67 \%$ of the intervention group and $70 \%$ of the control group; baseline data analysis indicated no difference between the groups based on child's age, gender, residence, number of siblings, family structure, caregiver's educational level, family asthma history, or disease duration. However, males were over represented in both groups which is consistent with the third nationwide survey of childhood asthma [6, 16]. The majority of families resided in urban areas and the majority of caregivers had a high school diploma with some secondary education. See Table 1.

\subsection{Asthma Self-Management and Frequency of PFM Use}

The total asthma self-management score and scores for its three dimensions, and the frequency of PFM use were not significantly different between the intervention and control groups at baseline. Total scores for self-management as well as for daily life, medication, and psycho-social management demonstrated statistically significant improvement in the intervention group. Scores for medicine management increased markedly from 56.78 to 75.78 . Significant changes in scores for total self-management $(P<0.001)$ and its three dimensions $(P<0.01 ; P<0.001 ; P<0.05$ respectively) were found between groups. See Table 2. Only one child among the 72 children used a PFM before participating in this study. The 2-month follow-up visit revealed that 29 children used the PFM 4-6 times a week and only one child in the intervention group never used the PFM; whereas 31 children in the control group never used the PFM. See Fig. 2. 
Table 1 Demographic characteristics of participants who completed data collection at baseline and 2-month follow-up by group assignment.

\begin{tabular}{|c|c|c|c|}
\hline & $\begin{array}{l}\text { Intervention Group }(n=36) \\
n(\%)\end{array}$ & $\begin{array}{l}\text { Control Group }(n=36) \\
n(\%)\end{array}$ & Chi-square between Groups \\
\hline \multicolumn{4}{|l|}{ Age in years } \\
\hline 9 & $15(41.7)$ & $11(30.6)$ & \multirow[t]{5}{*}{0.711} \\
\hline 10 & $9(25.0)$ & $14(38.9)$ & \\
\hline 11 & $6(16.7)$ & $5(13.9)$ & \\
\hline 12 & $3(8.3)$ & $4(11.1)$ & \\
\hline 13 & $3(8.3)$ & $2(5.6)$ & \\
\hline \multicolumn{4}{|l|}{ Gender } \\
\hline Male & $26(72.2)$ & $30(83.3)$ & \multirow[t]{2}{*}{0.257} \\
\hline Female & $10(27.8)$ & $6(16.7)$ & \\
\hline \multicolumn{4}{|l|}{ Residence } \\
\hline Urban-City & $27(75.0)$ & $30(83.3)$ & \multirow[t]{2}{*}{0.384} \\
\hline Rural-Country & $9(25.0)$ & $6(16.7)$ & \\
\hline \multicolumn{4}{|l|}{ Only child } \\
\hline Yes & $31(86.1)$ & $28(77.8)$ & \multirow[t]{2}{*}{0.358} \\
\hline No & $5(13.9)$ & $8(22.2)$ & \\
\hline \multicolumn{4}{|c|}{ Family structure in the home } \\
\hline Three generations & $15(41.7)$ & $15(41.7)$ & \multirow[t]{3}{*}{1.000} \\
\hline Two parents household & $19(52.8)$ & $19(52.8)$ & \\
\hline One parent household & $2(5.6)$ & $2(5.6)$ & \\
\hline \multicolumn{4}{|c|}{ Caregiver level of educational } \\
\hline Primary school & $4(11.1)$ & $4(11.1)$ & \multirow[t]{4}{*}{0.987} \\
\hline Junior high school & $6(16.7)$ & $5(13.9)$ & \\
\hline High school & $11(30.6)$ & $12(33.3)$ & \\
\hline College & $15(41.7)$ & $15(41.7)$ & \\
\hline \multicolumn{4}{|l|}{ Family asthma history } \\
\hline Yes & $12(33.3)$ & $7(19.4)$ & \multirow[t]{2}{*}{0.181} \\
\hline No & $24(66.7)$ & $29(80.6)$ & \\
\hline \multicolumn{4}{|c|}{ Disease duration since diagnosis } \\
\hline $1-2$ years & $5(13.9)$ & $7(19.4)$ & \multirow[t]{3}{*}{0.805} \\
\hline 3-4 years & $21(58.3)$ & $19(52.8)$ & \\
\hline More than 5 years & $10(27.8)$ & $10(27.8)$ & \\
\hline
\end{tabular}

Table 2 Changes in pediatric asthma management scores for children by intervention and control groups.

\begin{tabular}{|c|c|c|c|c|c|c|c|}
\hline & \multicolumn{3}{|c|}{ Intervention Group $(n=36)$} & \multicolumn{3}{|c|}{ Control Group $(n=36)$} & \multirow{2}{*}{$\begin{array}{l}P \text {-value } \\
\text { Change }\end{array}$} \\
\hline & $M(S D)$ & $M$ Change & $P$-value & $M(S D)$ & $M$ Change & $P$-value & \\
\hline Total self-management & $109.82(15.62)$ & 25.64 & $0.000^{* * *}$ & $108.50(19.54)$ & 2.28 & 0.241 & $0.000^{* * *}$ \\
\hline Daily life management & $21.33(4.01)$ & 3.47 & $0.000^{* * *}$ & $21.06(5.28)$ & 1.00 & 0.073 & $0.006^{* *}$ \\
\hline Medication management & $56.78(9.74)$ & 19.00 & $0.000^{* * *}$ & $56.00(10.73)$ & 0.69 & 0.592 & $0.000 * * *$ \\
\hline $\begin{array}{l}\text { Psychosocial } \\
\text { management }\end{array}$ & $31.69(5.86)$ & 3.17 & $0.001^{* *}$ & $31.44(6.20)$ & 0.58 & 0.301 & $0.013^{*}$ \\
\hline
\end{tabular}

${ }^{* *} P<0.01,{ }^{* *} P<0.001, \mathrm{M}=$ mean.

\subsection{Asthma Control and FEV1\%}

Asthma control scores and FEV1\% values were not significantly different between the intervention and control groups at baseline. However, the rate of well-controlled asthma for the intervention group dramatically increased over time from $13.89 \%$ to $77.78 \%$; whereas minimal change from $36.11 \%$ to $38.89 \%$ 

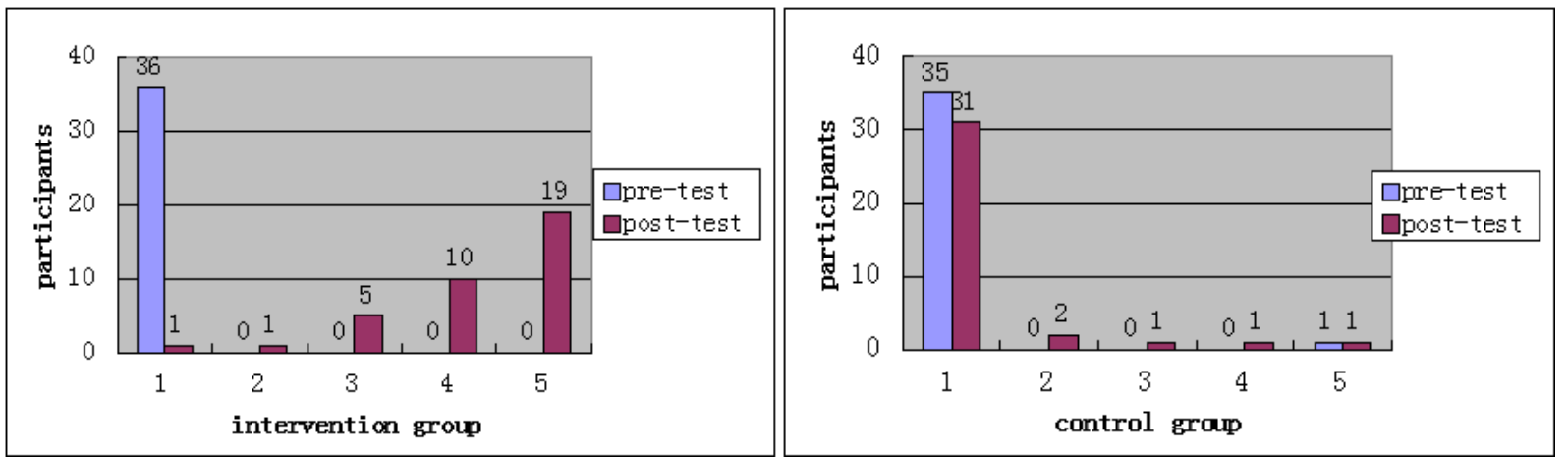

Fig. 2 PFM usage frequency at baseline and 2-month follow-up for the intervention and control groups.

For the X-axis labels, $1=$ never, $2=$ occasionally (1-2 times a week), $3=$ sometimes $(2-3$ times a week), $4=$ usually (4-6 times a week), and $5=$ always (every day) used PFM.

Table 3 Measures of asthma control for children at baseline and 2-month follow-up by intervention and control group.

\begin{tabular}{lllll}
\hline \multirow{2}{*}{ Asthma Control } & \multicolumn{2}{c}{ Baseline } & \multicolumn{2}{c}{ 2-month Follow-up } \\
\cline { 2 - 5 } & Intervention Group & Control Group & Intervention Group & Control Group \\
\hline Less-controlled $\geq 0.75$ & $n=31$ & $n=23$ & $n=8$ & $n=22$ \\
\hline Well-controlled $<0.75$ & $n=5$ & $n=13$ & $n=28$ & $n=14$ \\
Rate of well-controlled & 13.89 & 36.11 & 77.78 & 38.89 \\
\hline
\end{tabular}

Table 4 Changes in asthma control, child and caregiver quality of life, caregiver scores, and FEV1\% by intervention and control groups.

\begin{tabular}{llllllll}
\hline & \multicolumn{3}{c}{ Intervention Group $(n=36)$} & \multicolumn{2}{c}{ Control Group $(n=36)$} & $P$-value \\
\cline { 2 - 7 } & $M(S D)$ & Mean Change & $P$-value & $M(S D)$ & Mean Change & $P$-value & Change \\
\hline Asthma Control & $1.583(0.86)$ & -1.06 & $0.000^{* * *}$ & $1.214(0.46)$ & -0.22 & 0.167 & $0.000^{* * *}$ \\
\hline Child's quality of life & $4.990(1.16)$ & 0.85 & $0.000^{* * *}$ & $5.118(0.77)$ & 0.18 & 0.061 & $0.000^{* * *}$ \\
Activity limitation & $5.839(2.71)$ & 0.66 & 0.524 & $5.617(0.89)$ & -0.19 & 0.090 & $0.000^{* * *}$ \\
Symptoms & $4.461(1.15)$ & 1.21 & $0.000^{* * *}$ & $4.61(0.84)$ & 0.61 & $0.000^{* * *}$ & $0.008^{* *}$ \\
Emotional functioning & $5.122(0.81)$ & 0.82 & $0.000^{* * *}$ & $4.764(0.80)$ & 0.56 & $0.000^{* * *}$ & 0.158 \\
Caregiver quality of life & $3.579(0.93)$ & 1.14 & $0.000^{* * *}$ & $3.825(0.86)$ & 0.07 & 0.355 & $0.000^{* * *}$ \\
Activity limitation & $3.618(1.12)$ & 1.22 & $0.000^{* * *}$ & $3.861(1.06)$ & 0.12 & 0.254 & $0.000^{* * *}$ \\
Emotional functioning & $3.562(0.96)$ & 1.10 & $0.000^{* * *}$ & $3.809(0.90)$ & 0.06 & 0.537 & $0.000^{* * *}$ \\
Pulmonary Function & $82.67(16.63)$ & 11.19 & $0.000^{* * *}$ & $84.11(17.59)$ & 6.31 & 0.069 & 0.234 \\
FEV1\% & & & &
\end{tabular}

${ }^{* *} P<0.01,{ }^{* *} P<0.001, \mathrm{M}=$ mean.

were found in the control group. See Table 3. FEV1\% values were not statistically or clinically different at the 2-month follow-up appointment. See Table 4.

\subsection{Child and Caregiver Quality of Life}

No significant differences in child or caregiver quality of life between the intervention and control group were observed at baseline. Quality of life scores for children assigned to the intervention group demonstrated statistically significant improvement $(P$ $<0.001)$ in total quality of life as well as in symptoms and emotional function, but not for activity limitation. Scores for children assigned to the control group demonstrated significant improvement $(P<0.001)$ in symptoms and emotional function, but not total quality of life or activity limitation. All scores except those for emotional functioning in the intervention group showed significant improvement $(P<0.01)$ when compared to the control group.

Quality of life scores for caregivers assigned to the intervention group demonstrated statistically significant improvement $(P<0.001)$ in total quality of 
life as well as activity limitation and emotional function; whereas scores for caregivers assigned to the control group failed to reach clinical or statistical significance. The changes at pre and posttest between the two groups demonstrated significant improvement $(P<0.001)$ in quality of life in those caregivers. See Table 4.

\section{Discussion}

The aim of this study was to evaluate the efficacy of an outpatient asthma self-management education intervention for children with asthma and their caregiver in Wuhan, China. Findings supported the efficacy and clinical significance of the intervention based on increased asthma self-management scores, increased frequency in PFM use, increased asthma control level, and increased child and caregiver quality of life scores in the intervention group.

This study was undertaken because of the lack of community health care resources in China [24] and the inadequacy of asthma education by physicians, especially for children with asthma [25]. The present study used quality of life, level of asthma control, frequency of PFM usage and pulmonary function suggested by Fredric Wolf [26] to evaluate outcomes rather than emergency department visits, hospitalization and missed school days, which have been extensively used.

\subsection{Asthma Self-management and Frequency of PFM Use}

The finding of significant improvement on the PASMS in the intervention group was consisted with the findings of Tao, who found that the use of home-basic asthma intervention improved the self-management of asthma among children aged 7 to 17 [27]. Health education related to asthma in the intervention group could explain this change. Basic knowledge of asthma treatment is important for children and their caregivers/parents [25] especially in those having severe asthma to increase compliance with steroid therapy and inhaler skills. The dramatic increase in the medication management dimension $(\mathrm{d}=$ 19.00) of the SMSPA in the present study can indicate better asthma control for those children.

In the third national survey of asthma in China, $14.3 \%$ of children were reported using a PFM to monitor their symptoms [6]. However, most of the participants in this study did not previously use a PFM. After intervention, more than three fourths of participants in the intervention group used a PFM to self-monitor asthma symptoms. Since there are proven benefits in PFM usage [7], health providers in the clinical setting should recommend this self-monitoring action.

\subsection{Asthma Control, Pulmonary Function and Quality of Life}

The health outcomes used in this study including level of asthma control, scores on the PAQLQ and PACQLQ, and the results of the FEV1\% showed improved in both the intervention and control groups. Similar to previous studies [2], asthma control level improved after intervention. While in this study, the rate of well-controlled asthma management increased from $13.9 \%$ to $77.8 \%$ in the intervention group compared with $36.1 \%$ to $38.9 \%$ in the control group. Furthermore, increased medical adherence, better understanding of steroid therapy, and improved quality of life are perceived benefits of this asthma self-management intervention. Findings also suggest that acquisition of the self-monitoring skills to achieve better asthma control according to use of the PFM or symptoms may reduce the activity limitations of children.

Although FEV1\% values were higher in the intervention group than in the control group, no significant difference between the groups was found which was consistent with Rasberry's study [28]. On the contrary, in an outpatient education program for children with uncontrolled asthma $\geq 14$ years of age, FEV1\% was proved to be significantly increased [2]. The different characteristics of the participants, the 
small sample size, varying asthma education content and the interval to retest FEV1\% may have led to these results. Additionally, techniques required by PFT may be beyond some children' abilities.

\subsection{Limitations}

Limitations in the present study include small sample size and sampling from only one hospital. These may introduce selection bias. Other potential limitations may occur due to the lack of experience of the children in providing precise answers to survey items for children aged 9-13. Although the 2-month follow up showed a significant improvement in self-management competence of the children, further follow up in recommended to evaluate the effects of this program.

\subsection{Implications for Clinical Practice}

The increasing incidence of pediatric asthma in China poses a need for nurses to make a change in asthma management of children. Lacking knowledge and misunderstanding of the required therapies are the main reasons for substandard asthma treatment $[8,29$, 30]. Therefore, it is important to educate nurses in China about proper asthma management for children. In addition, clinical protocols for asthma management among children should be put into place.

Favorable results in the present study are powerful evidence that asthma education can improve knowledge and impel self-management behaviors which are indispensable to keeping asthma well-controlled and improving compliance to medication therapy. Nurses in departments that provide asthma care should explore various formats of self-management education to achieve improved health outcomes. On one hand, community hospitals are the mainstay of the Chinese health system in the future as recommended by the Chinese government. On the other hand, the lack of nurses qualified in asthma management in community hospitals [24] prohibits the implementation of effective asthma education programs. Education programs for Nurses should include asthma self-management knowledge and practical interventions which are specific to urban and rural health care settings.

\subsection{Suggestions for Advancing Health Policy}

The results of the present study indicate the necessity and importance for conducting asthma education among affected children, and the need for follow-up care.

\subsection{Recommendations for Future Research}

Future studies should include a larger sample size and increase the number of sessions on asthma education. Additional research is needed to compare the effects of asthma education in diverse settings. More economic self-management methods should be explored as these would improve efficiency of health services delivery in China.

\section{Conclusions}

Limited research in China has examined the efficacy of self-management among children with asthma. The present study provides a practical and effective method for health care providers to improve asthma self-management, PFM usage frequency, asthma control level, and the quality of life among both children and their main caregivers. For positive outcomes, education about self-management of asthma among children should be a standard of care in China.

\section{Acknowledgments}

The authors would like to thank professor Juniper and Dr. Ping $\mathrm{Cu}$ for kindly sharing their scales; all participants for joining and completing this study.

\section{References}

[1] Bousquet, J., Mantzouranis, E., Cruz, A. A., Ait-Khaled, N., Baena-Cagnani, C. E., Bleecker, E. R., Brightling, C. E., Burney, P., Bush, A., Busse, W. W., Casale, T. B., Chan-Yeung, M., Chen, R., Chowdhury, B., Chung, K. F., Dahl, R., Drazen, J. M., Fabbri, L. M., Holgate, S. T., Kauffmann, F., Haahtela, T., Khaltaev, N., Kiley, J. P., 

with Asthma and Their Caregiver in Wuhan, China

Masjedi, M. R., Mohammad, Y., O’Byrne, P., Patridge, M. R., Rabe, K. F., Togias, A., Weel, C. V., Wenzel, S., Zhong, N., and Zuberbier, T. 2008. "Uniform Definition of Asthma Severity, Control, and Exacerbations: Document Presented for the World Health Organization Consultation on Severe Asthma." J. Allergy Clin. Immunol. 126 (5): 926-38.

[2] Rodrigues, C. D., Pereira, R. P., and Dalcin, P. T. 2013. "Effects of an Outpatient Education Program in Patients with Uncontrolled Asthma." J. Bras. Pneumol. 39 (3): 272-9.

[3] Lahaye, M., Luminet, O., Van, B. N., and Bodart, E. 2012. "Psychological, Social and School Implications of Asthma: A Comparison of Belgian French-Speaking Children Having Asthma with Healthy Children." Acta Clinica Belgica 67 (1): 25-9.

[4] Bai, Y., Hillemeier, M. M., and Lengerich, E. J. 2007. "Racial/Ethnic Disparities in Symptom Severity among Children Hospitalized with Asthma." J. Health Care Poor Underserved 18 (1): 54-61.

[5] Akinbami, L. J., Moorman, J. E., Garbe, P. L., and Sondik, E. J. 2009. "Status of Childhood Asthma in the United States, 1980-2007." PEDIATRICS 123 (Suppl 3): S131-45.

[6] The National Cooperative Group on Childhood Asthma, Institute of Environmental Health and Related Product Safety, Chinese Center for Disease Control and Prevention. 2013. "The Third Nationwide Epidemiology Survey of Childhood Asthma in Urban Areas." Chinese Journal of Pediatrics 51 (10): 729-35. (in Chinese)

[7] Reddel, H. K., and Levy, M. L. 2015. "The GINA Asthma Strategy Report: What's New for Primary Care?" NPJ Prim. Care Respir. Med. 25: 15050.

[8] Gao, G. Z., Li, G. F., and Liu, W. J. 2011. "The Qualitative Research on Status of Asthma Self-management and Nursing Needs." Chinese Journal of Modern Nursing 17 (34): 4121-2. (in Chinese)

[9] McQuaid, E. L., Garro, A., Seifer, R., Hammond, S. K., and Borrelli, B. 2012. "Integrating Asthma Education and Smoking Cessation for Parents: Financial Return on Investment." Pediatr. Pulm. 47 (10): 950-5.

[10] Findley, S. E., Thomas, G., Madera-Reese, R., McLeod, N., Kintala, S., Andres Martinez, R., Ortiz, B., and Herman, E. 2011. "A Community-Based Strategy for Improving Asthma Management and Outcomes for Preschoolers." Journal of Urban Health 88 (S1): 85-99.

[11] Turyk, M., Banda, E., Chisum, G., Weems, D., Liu, Y., Damitz, M., Williams, R., and Persky, V. 2013. "A Multifaceted Community-Based Asthma Intervention in Chicago: Effects of Trigger Reduction and Self-management Education on Asthma Morbidity." J. Asthma. 50 (7): 729-36.
[12] Kintner, E., Cook, G., Marti, C. N., Stoddard, D., Gomes, M., Harmon, P., and Van Egeren, L. A. 2015. "Comparative Effectiveness on Cognitive Asthma Outcomes of the SHARP Academic Asthma Health Education and Counseling Program and a Non-Academic Program.” Res. Nurs. Health 38 (6): 423-35.

[13] Nelson, K. A., Highstein, G. R., Garbutt, J., Trinkaus, K., Fisher, E. B., Smith, S. R., and Strunk, R. C. 2011. "A Randomized Controlled Trial of Parental Asthma Coaching to Improve Outcomes among Urban Minority Children." Archives of Pediatrics \& Adolescent Medicine 165 (6): 520-6.

[14] Horner, S. D., and Brown, A. 2014. "Evaluating the Effect of an Asthma Self-Management Intervention for Rural Families." J. Asthma. 51 (2): 168-77.

[15] Juniper, E. F., O’Byrne, P. M., Guyatt, G. H., Ferrie, P. J., and King, D. R. 1999. "Development and Validation of a Questionnaire to Measure Asthma Control." Eur. Respir. J. 14 (4): 902-7.

[16] Chinese Medical Association, The Subspecialty Group of Respiratory Diseases of Pediatrics. 2008. "Guidelines on Diagnosis and Therapy for Children with Asthma in 2008." Chinese Journal of Pediatrics 46 (10): 745-53. (in Chinese)

[17] Expert Panel Report 3 (EPR-3). 2007. "Guidelines for the Diagnosis and Management of Asthma-Summary Report 2007." J. Allergy Clin. Immunol. 120 (5 Suppl.): S94-138.

[18] Hu, P., Zhang, J., Huang, Y., Yuan, X. P., and Wang, Q. 2010. "Development and Validation of Pediatric Asthma Self-management Scale (PASMS)." Chinese General Practice 5 (31): 3513-5. (in Chinese)

[19] Juniper, E. F., O’Byrne, P. M., Guyatt, G. H., Ferrie, P. J., and King, D. R. 1999. "Development and Validation of a Questionnaire to Measure Asthma Control." European Respiratory Journal 14 (4): 902-7.

[20] Fuhlbrigge, A. L., Kitch, B. T., Paltiel, A. D., Kuntz, K. M., Neumann, P. J., Dockery, D. W., and Wess, S. T. 2001. "FEV(1) Is Associated with Risk of Asthma Attacks in a Pediatric Population.” J. Allergy Clin. Immunol. 107 (1): 61-7.

[21] Juniper, E. F., Buist, A. S., Cox, F. M., Ferrie, P. J., and King, D. R. 1999. "Validation of a Standardized Version of the Asthma Quality of Life Questionnaire." Chest 115 (5): 1265-70.

[22] Juniper, E. F., Guyatt, G. H., Feeny, D. H., Ferrie, P. J., Griffith, L. E., and Townsend, M. 1996. "Measuring Quality of Life in Children with Asthma." Qual. Life Res. 5 (1): 35-46.

[23] Cerdan, N. S., Alpert, P. T., Moonie, S., Cyrkiel, D., and Rue, S. 2012. "Asthma Severity in Children and the Quality of Life of Their Parents" Appl. Nurs. Res. 25 (3): 131-7. 


\section{with Asthma and Their Caregiver in Wuhan, China}

[24] Xi, F. J., Tang, Q., Wang, R., Zhang, T. T., Gao, R., and Jiang, Y. L. 2015. "The Status and Prospect of Community Nursing in China." Today Nurse 1 (2): 1-3. (in Chinese)

[25] Orrell-Valente, J. K., Jones, K., Manasse, S., Thyne, S. M., Shenkin, B. N., and Cabana, M. D. 2011. "Children's and Parents' Report of Asthma Education Received from Physicians." J. Asthma. 48 (8): 831-8.

[26] Wolf, F. M., Guevara, J. P., Grum, C. M., Clark, N. M., and Cates, C. J. 2003. "Educational Interventions for Asthma in Children." Cochrane Database Syst. Rev. (1): D326.

[27] Tao, M. Y. 2013. "The Impact of Systematic Family Intervention on the Self-management of Asthma Children and the Mental Health of Parents." University of South China. Accessed July 30, 2015. http://www.cnki.net/KCMS/detail/detail.aspx?FileName= 1013341285.nh\&DbName=CMFD2014 (in Chinese)
[28] Rasberry, C. N., Cheung, K., Buckley, R., Dunville, R., Daniels, B., Cook, D., Robin, L., and Dean, B. 2014. "Indicators of Asthma Control among Students in a Rural, School-Based Asthma Management Program.” J. Asthma. 51 (8): 876-85.

[29] Koster, E. S., Wijga, A. H., Koppelman, G. H., Postma, D. S., Brunekreef, B., DeJongste, J. C., Smit, H. A., Hoekstra, M. O., Raaijmakers, J. A., and Maitland-Van, Z. A. 2011. "Uncontrolled Asthma at Age 8: The Importance of Parental Perception towards Medication." Pediatr. Allergy Immunol. 22 (5): 462-8.

[30] Al-Muhsen, S., Dulgom, S., Assiri, Z., Al-Jahdali, H., Horanieh, N., Vazquez-Tello, A., and Al-Jahdali, H. 2015. "Poor Asthma Education and Medication Compliance Are Associated with Increased Emergency Department Visits by Asthmatic Children." Ann. Thorac. Med. 10 (2): 123. 